\title{
Effect of iterative reconstruction algorithm levels on noise index and figure-of-merit in CT pulmonary angiography examinations
}

\begin{abstract}
Purpose: To evaluate the influence of iterative reconstruction (IR) levels on Computed Tomography (CT) image quality and to establish Figure of Merit (FOM) value for CT Pulmonary Angiography (CTPA) examinations. Methods: Images of 31 adult patients who underwent CTPA examinations in our institution from March to April 2019 were retrospectively collected. Other data, such as scanning parameters, radiation dose and body habitus information from the subjects were also recorded. Six different levels of IR were applied to the volume data of the subjects. Five circles of the region of interest (ROI) were drawn in five different arteries namely, pulmonary trunk, right pulmonary artery, left pulmonary artery, ascending aorta and descending aorta. The mean Signal-to-noise ratio (SNR) was obtained, and the FOM was calculated in a fraction of the SNR2 divided by volume-weighted CT dose index (CTDIvol) and SNR2 divided by the sizespecific dose estimates (SSDE). Results: Overall, we observed that the mean value of CTDIvol and SSDE were 13.79 $\pm 7.72 \mathrm{mGy}$ and $17.25 \pm 8.92 \mathrm{mGy}$, respectively. Notably, SNR values significantly increase with increase of the IR level $(\mathrm{p}<0.05)$. There are also significant differences $(\mathrm{p}<0.05)$ in the FOM for both SNR2/SSDE and SNR2/CTDIvol attained in different IR levels. Conclusion: We successfully evaluate the value of radiation dose and image quality performance and set up a figure of merit for both parameters to further verify scanning protocols by radiology personnel.
\end{abstract}

Keyword: Signal-to-noise ratio; Figure-of-merit; CT pulmonary angiography; Pulmonary embolism; Iterative reconstruction algorithm 